\title{
Benardete's Paradox and the Logic of Counterfactuals
}

\author{
Michael Caie*
}

In Benardete [1964], José Benardete presents the following puzzling scenario:

A man decides to walk one mile from A to B. A god waits in readiness to throw up a wall blocking the man's further advance when the man has travelled $1 / 2$ mile. A second god (unbeknownst to the first) waits in readiness to throw up a wall of his own blocking the man's further advance when the man has travelled $1 / 4$ mile. A third god ... etc. ad infinitum. It is clear that this infinite sequence of mere intentions (assuming the contrary-to-fact conditional that each god would succeed in executing his intentions if given the opportunity) logically entails the consequence that the man will be arrested at point A; he will not be able to pass beyond it, even though not a single wall will in fact be thrown down in his path. The ... [effect] will be described by the man as a strange field of force blocking has passage forward. ${ }^{1}$

This puzzling case has been discussed by a few authors. ${ }^{2}$ I think, however, that there is still a paradox lurking in this scenario that has not been clearly isolated. In this note, I will outline this paradox and suggest some lessons that may be drawn from it.

\section{The Paradox}

It will help if we describe the scenario that Benardete outlines in a bit more detail, and if we also engage in some selective redescription and relabeling.

Let us imagine a two-dimensional world with the metric structure of the Euclidean plane. We assume that a creature - call it $M$-is able to move along a line within this plane, and that it must do so in a continuous manner. Given a metric preserving mapping from the points of this line onto the real numbers, we associate each point with the corresponding real number. We assume that $M$ begins at some point $\mathbf{s}<0$ and moves toward 0 . For each $n \in \mathbb{N}$, and each point $\frac{1}{n}$, there is a god at that point who intends to throw up an impenetrable

*University of Pittsburgh / caiemike@gmail.com / Thanks to Fabrizio Cariani, Cian Dorr and Paolo Santorio for helpful discussion related to this paper.

${ }^{1}$ Emphasis added.

${ }^{2}$ See, for example, Priest [1999], Yablo [2000], Hawthorne [2000] and Uzquiano [2012]. 
(one-dimensional) wall at $\frac{1}{n}$, if $M$ makes it past $\frac{1}{n+1}$. Were such a wall to be thrown up at $\frac{1}{n}, M$ would not make it past this point. We assume, further, that there are no walls at any point $z \leq 0$ and, finally, that $M$ 's progress will remain unimpeded unless there is a wall to stop it.

It will help to have some definitional abbreviations on hand.

Def. Let $P_{z}={ }_{d f} M$ has passed $z$, i.e., has made it to a point $q>z$.

Def. Let $W_{z}={ }_{d f}$ there is an impenetrable wall at point $z .^{3}$

The first thing to note is that, given the description of the case, it would seem that if $M$ were to make it past $\frac{1}{n+1}$, then the god at $\frac{1}{n}$ would throw up a wall at $\frac{1}{n}$, and $M$ would not make it past $\frac{1}{n}$. That is, given the description of the case, it would seem that we have each instance of the following counterfactual schema:

Intention Realization $:_{n} P_{\frac{1}{n+1}} \square \rightarrow\left(W_{\frac{1}{n}} \wedge \forall_{z \leq n} \neg P_{\frac{1}{z}}\right)$

And so, given the description of the case, it would seem that we have the following infinite conjunction:

$$
\text { Intention Realization: } \bigwedge_{n \in \mathbb{N}}\left[P_{\frac{1}{n+1}} \square \rightarrow\left(W_{\frac{1}{n}} \wedge \forall_{z \leq n} \neg P_{\frac{1}{z}}\right)\right]
$$

But, given the description of the case, we also have ${ }^{4}$ :

Only Walls: $\forall_{y}\left[\left(\neg \exists_{x \leq y} W x\right) \rightarrow P y\right]$

No Walls: $\neg \exists_{x \leq 0} W x$

The problem, though, is that, given very minimal assumptions about the logic of counterfactuals, we have that Intention Realization, Only Walls, and No Walls are jointly inconsistent. While, then, it would seem like we can describe a coherent situation in which all of these claims hold, this turns out to be false.

Claim: Intention Realization, Only Walls, and No Walls are jointly inconsistent.

Justification: We first show that, given Intention Realization, we have $\neg P_{\frac{1}{n}}$, for each $n \in \mathbb{N}$. Assume, then, for arbitrary $n \in \mathbb{N}$, that $P_{\frac{1}{n}}$. Then, since if $M$ has passed $\frac{1}{n}, M$ has also passed $\frac{1}{n+1}$, we have $P_{\frac{1}{n+1}}$. Given Intention Realization, though, and the minimal assumption that the inference from $\phi \square \hookrightarrow \psi$ and $\phi$ to $\psi$ is valid, it follows from $P_{\frac{1}{n+1}}$ that we have $\forall_{z \leq n} \neg P_{\frac{1}{z}}$. And so, in particular,

\footnotetext{
${ }^{3}$ Strictly speaking, of course, both of these should be time-indexed. However, to avoid distracting clutter, we'll leave it to context to determine the appropriate time parameter.

${ }^{4}$ Here ' $\rightarrow$ ' should be read as the material conditional.
} 
we have $\neg P_{\frac{1}{n}}$, which, together with $P_{\frac{1}{n}}$, gives us a contradiction. By reductio, then, we have $\neg P_{\frac{1}{n}}$. And, since $n$ was arbitrary, it follows that we have, for each $n \in \mathbb{N}, \neg P_{\frac{1}{n}}$.

Given Only Walls and No Walls, however, it follows that, for some $n \in \mathbb{N}, P_{\frac{1}{n}}$. For Only Walls and No Walls together entail $P_{0}$. But, of course, if $M$ makes it to some point $z>0$, then it follows that there is some $n \in \mathbb{N}$, such that $M$ makes it to some point $z>\frac{1}{n}$.

And so we have that, for some $n \in \mathbb{N}, P_{\underline{1}}$, which together with the claim that, for each $n \in \mathbb{N}, \neg P_{\frac{1}{n}}$, gives us a contradiction.

Consider now the following two claims:

Intention Realization Possibility: There is some metaphysically possible scenario in which Intention Realization holds.

Conditional Compossibility: If Intention Realization is metaphysically possible, then it is metaphysically compossible with Only Walls and No Walls.

Both of these claim are, I think, at least prima facie plausible.

On the one hand, Intention Realization Possibility is prima facie plausible. For it certainly seems like, in the scenario described, each god should be able to realize their intention were they called upon to do so. To drive this home we could flesh out the situation in greater detail. We might, for example, imagine that when such gods are not arranged in such a sequence they are able to call up such a wall at will. Why, then, would they not all be able to realize their intentions, were they called upon to do so, given that they are arranged as described? At first glance, at least, it isn't obvious what a good answer to this question would be.

On the other hand, Conditional Compossibility is also prima facie plausible. For the facts that would seem to ensure that Intention Realization holds, namely the facts about the gods' intentions to throw up walls at various points past 0 , were $M$ to make it past other points beyond 0 , and their general efficaciousness, wouldn't seem to be the sorts of facts that constrain what might happen to $M$ at points prior to and including 0 .

However, despite the fact that both Intention Realization Possibility and Conditional Compossibility are prima facie plausible, the preceding shows that one of these claims must be rejected. Either Intention Realization isn't metaphysically possible, or it is metaphysically possible but the situations in which Intention Realization hold are all such that either Only Walls or No Walls fail. ${ }^{5}$

\footnotetext{
${ }^{5}$ It would take us too far afield to consider in detail how this paradox relates to those versions already formulated in the literature. The key difference, however, is that other discussions of Benardete's scenario don't formulate the paradox that it is supposed to generate in terms of counterfactuals. Thus, Priest [1999] and Yablo [2000] consider a paradox formulated in terms of material conditionals, while Hawthorne [2000] considers one formulated in terms of causal laws. These paradoxes are certainly all interesting in their own right. However, I think that the paradox isolated here raises interesting issues that don't arise in these other versions that do not explicitly involve counterfactuals. And, for what it's worth, given the explicit appeal to counterfactuals in the presentation of this puzzling scenario, it seems natural to consider whether there might be a paradox generated by this scenario that explicitly involves counterfactual claims.
} 


\section{$2 \quad$ Rejecting Conditional Compossibility.}

Let's first consider the option of endorsing Intention Realization Possibility and rejecting Conditional Compossibility. According to this view, at least in some worlds in which something impedes $M$ 's progress before it reaches 0 , each of the gods is such that it would realize its intention to block $M$ by throwing up a wall, were it called upon to do so. This, indeed, would seem to be the view endorsed by Benardete.

We can distinguish two views within this camp.

On the one hand, one might maintain that the sorts of facts that would seem to support Intention Realization - namely the gods' intentions and the individual facts about them that ensure that they are typically able to realize their intentions to create walls - suffice to ensure that Intention Realization holds, as long as something impedes $M$ 's progress at or before $0 .{ }^{6}$

On the other hand, one might deny that every case in which the gods have the appropriate intensions etc. and in which $M$ 's progress is halted is one in which Intention Realization holds. Instead, one might maintain that Intention Realization will hold, if, but only if, in addition to the gods' intentions etc. it is a causal law that such intentions etc. serve to prevent $M$ 's passage beyond $0 .^{7}$

Now I don't think that either of these views is incoherent. Both, however, have the surprising consequence that while the intentions of the gods and their general efficacy do not ensure that Intention Realization holds, these facts, in conjunction with some other facts that, at least initially might seem to be irrelevant to the counterfactuals that are the conjuncts of Intention Realization, do ensure that Intention Realization holds.

For example, at least initially, it is hard to see why, if the intentions of the gods and their general efficacy do not themselves suffice to ensure that Intention Realization holds, the addition of some impediments, causally unconnected to the intentions of the gods, that arise at or before 0 should, together with those intentions, suffice to ensure that Intention Realization holds. For Intention Realization concerns what would happen at various points beyond 0 , were $M$ to make it to other points beyond 0 .

And, at least initially, it is also hard to see why, if the intentions of the gods and their general efficacy do not themselves suffice to ensure that Intention Realization holds, the addition of causal laws that dictate that the gods' intentions to throw up walls at various points after 0 causally suffice to ensure that some event stops $M$ at or before 0 should, together with those intentions, suffice to ensure that Intention Realization holds. For, at least initially, what would seem to make the intentions of the gods and their general efficacy suffice to ensure that Intention Realization holds is simply the potential causal connection between such intentions and walls that might be built at various points after 0 .

Each of the above views, though, would have us reject one of these initially plausible thoughts. Now perhaps the lesson to draw from this paradox is that we should reject one of these plausible thoughts. I'm inclined to think, though, that the plausibility of the claim

\footnotetext{
${ }^{6}$ Although he does not frame the puzzle raised by Benardete's scenario in terms of counterfactuals, one can perhaps see something akin to this sort of view in Yablo [2000].

${ }^{7}$ Although he also does not frame the puzzle raised by Benardete's scenario in terms of counterfactuals, one can perhaps see something akin to this sort of view in Hawthorne [2000].
} 
that whether Intention Realization holds, given the intentions of the gods etc., should be independent of whether there is anything that stops $M$ at or before 0, gives us good reason to think that the lesson to draw from this paradox is that, despite appearances, the intentions of the gods and their general efficacy couldn't suffice to ensure that Intention Realization holds, even in conjunction with other facts concerning what happens to $M$ at or before 0 . Furthermore, I think that there are general principled grounds for maintaining this.

\section{$3 \quad$ Rejecting Intention Realization Possibility}

Let's now consider the option of rejecting Intention Realization Possibility. To see why there are principled grounds for rejecting this claim, despite its prima facie plausibility, consider the following:

Intention Failure: $\bigvee_{n \in \mathbb{N}} P_{\frac{1}{n+1}} \square \rightarrow \bigvee_{n \in \mathbb{N}}\left[P_{\frac{1}{n+1}} \wedge \neg\left(W_{\frac{1}{n}} \wedge \forall_{z \leq n} \neg P_{\frac{1}{z}}\right)\right]$

This says, roughly, that if $M$ were to make it some distance past 0 , then, for some $n \in \mathbb{N}$, $M$ would make it past $\frac{1}{n+1}$ and it would not be the case that the god at $\frac{1}{n}$ would throw up a wall at $\frac{1}{n}$ and $M$ would not make it past $\frac{1}{n}$.

Claim: Intention Failure holds at every possible world.

Justification: To see why this holds, note that Intention Failure follows from: ${ }^{8}$

(*) $\bigvee_{n \in \mathbb{N}} P_{\frac{1}{n+1}} \square \rightarrow \bigvee_{n \in \mathbb{N}}\left[P_{\frac{1}{n+1}} \wedge \exists_{z \leq n} P_{\frac{1}{z}}\right]$

But $(*)$ is guaranteed to hold at any possible world. For, given any $n+1$, if $M$ has made it past $\frac{1}{n+1}$, there is some $m>n$, such that $M$ has made it past $\frac{1}{m+1}$ and also past $\frac{1}{z}$, for some $z \leq m$. Since, then, Intention Failure follows from $(*)$, it follows that Intention Failure holds at every possible world.

${ }^{8}$ Justification: Note first that:

$$
\bigvee_{n \in \mathbb{N}} P_{\frac{1}{n+1}} \square \longrightarrow \bigvee_{n \in \mathbb{N}}\left[P_{\frac{1}{n+1}} \wedge \neg\left(W_{\frac{1}{n}} \wedge \forall_{z \leq n} \neg P_{\frac{1}{z}}\right)\right]
$$

is equivalent to:

$$
\bigvee_{n \in \mathbb{N}} P_{\frac{1}{n+1}} \square \rightarrow \bigvee_{n \in \mathbb{N}}\left[P_{\frac{1}{n+1}} \wedge\left(\neg W_{\frac{1}{n}} \vee \exists_{z \leq n} P_{\frac{1}{z}}\right)\right]
$$

And the latter clearly follows from:

$$
\bigvee_{n \in \mathbb{N}} P_{\frac{1}{n+1}} \square \rightarrow \bigvee_{n \in \mathbb{N}}\left[P_{\frac{1}{n+1}} \wedge \exists_{z \leq n} P_{\frac{1}{z}}\right]
$$


Next consider the following: ${ }^{9}$

Possible Passage: $\bigwedge_{n \in \mathbb{N}} \diamond P_{\frac{1}{n+1}}$

Claim: Possible Passage holds at every possible world.

Justification: Even if, for each $n \in \mathbb{N}$, it is impossible, given the constraints imposed on the putative possible world described by Benardete's scenario, for $M$ to make it past $\frac{1}{n+1}$, we surely shouldn't maintain that it is metaphysically impossible for $M$ to make it past $\frac{1}{n+1}$. For, of course, there are plenty of possible worlds in which $M$ travels along the appropriate line and these constraints are absent. For each $n \in \mathbb{N}$, then, there should be some possible world in which $M$ makes it past $\frac{1}{n+1}$. Thus, for any possible world, and each $n \in \mathbb{N}$, it will be metaphysically possible for $M$ to make it past $\frac{1}{n+1}$. And so, at each possible world, Possible Passage will hold.

Now, at a certain level of abstraction, we can see Intention Realization, Intention Failure, and Possible Passage as having the following logical forms:

$$
\begin{aligned}
& \text { (ILF1): } \bigwedge_{n \in \mathbb{N}}\left(\phi_{n} \square \rightarrow \psi_{n}\right) \\
& \text { (ILF2): } \bigvee_{n \in \mathbb{N}} \phi_{n} \square \rightarrow \bigvee_{n \in \mathbb{N}}\left(\phi_{n} \wedge \neg \psi_{n}\right) \\
& \text { (ILF3): } \bigwedge_{n \in \mathbb{N}} \diamond \phi_{n}
\end{aligned}
$$

There are, however, good grounds, I think, for maintaining that any three claims of the form (ILF1) - (ILF3) are jointly inconsistent. Assuming that this is so, it follows that we have good grounds for rejecting Intention Realization Possibility. For, if any claims of the form (ILF1) - (ILF3) are jointly inconsistent, then it follows that Intention Realization, Intention Failure, and Possible Passage are jointly inconsistent. However, we've argued that both Intention Failure and Possible Passage hold at every possible world. Given this, though, it follows that, if, given the logic of counterfactuals, Intention Realization, Intention Failure, and Possible Passage are inconsistent, then Intention Realization will fail to hold at any possible world, and so we must reject Intention Realization Possibility.

To see why it's plausible that a reasonable logic for counterfactuals will tell us that any three claims of the form (ILF1), (ILF2), and (ILF3) are jointly inconsistent, consider, first, the following set of schemas:

\footnotetext{
${ }^{9}$ I'll understand $\diamond \phi$ to mean that $\phi$ is metaphysically possible. In what follows, I'll assume that metaphysical possibilities do not lead to absurdity given counterfactual supposition. Thus, if we have $\diamond \phi$, then it isn't the case that, for some $\psi, \phi \square \mapsto \psi$ and $\phi \square \mapsto \neg \psi$ both hold. This fact will be tacitly appealed to at various points in what follows, for example at certain points in which it is shown that claims that have the logical form of Intention Realization, Intention Failure, and Possible Passage are inconsistent given certain semantic treatments of the counterfactual. If one is skeptical of the general claim that metaphysical possibilities do not lead to absurdity given counterfactual supposition, then simply substitute for Possible Passage the claim that, for each $n \in \mathbb{N}, P_{\frac{1}{n+1}}$ is coherently counterfactually supposible. This seems to me obvious, and, given this alternative assumption, the arguments can proceed mutatis mutandis.
} 
(1) $\phi \square \mapsto \psi$

(2) $\chi \square \mapsto \xi$

(3) $(\phi \vee \chi) \square \leftrightarrow(\phi \wedge \neg \psi) \vee(\chi \wedge \neg \xi)$

(4) $\diamond \phi \wedge \diamond \psi$

Now, I think that intuitively claims of the form (1) - (4) are inconsistent. For, given (3), it would seem that we should conclude that either things would not be as (1) claims they would be were $\phi$ to be the case, or things would not be as (2) claims they would be were $\chi$ to be the case. But, then, (1), (2) and (3) should be inconsistent, at least given the further assumption, provided by (4), that $\phi$ and $\psi$ are possible and so are coherently counterfactually supposible.

To see this, it will perhaps help to consider a particular instantiation of these schemas. Imagine, then, that Joshua and Aparna are playing a quiz game with some friends and are both asked a question. Now consider the following set of claims:

$\left(1^{*}\right)$ If Joshua were to answer, he'd get it right.

$\left(2^{*}\right)$ If Aparna were to answer, she'd get it right.

$\left(3^{*}\right)$ If either were to answer, at least one would get it wrong.

$\left(4^{*}\right)$ For both Joshua and Aparna, it is metaphysically possible for them to answer.

Now, $\left(4^{*}\right)$ obviously holds. So, with this in the background, focus on $\left(1^{*}\right)-\left(3^{*}\right)$. To my ear, at least, these sound obviously contradictory. If both would get the answer right, were they to answer, then if either were to answer, it seems clear that neither would get the answer wrong. But, given $\left(4^{*}\right)$, this should contradict $\left(3^{*}\right)$.

The intuition that claims of the form (1) - (4) are inconsistent should, I think, generalize quite naturally. Thus, the same sorts of considerations that motivate the claim that (1) - (4) are inconsistent should motivate the claim that counterfactuals of the following form are inconsistent:

$$
\begin{aligned}
& \text { (FLF1): } \bigwedge_{n \in\{1,2, \ldots m\}}\left(\phi_{n} \square \mapsto \psi_{n}\right) \\
& \text { (FLF2): } \bigvee_{n \in\{1,2, \ldots m\}} \phi_{n} \square \rightarrow \bigvee_{n \in\{1,2, \ldots m\}}\left(\phi_{n} \wedge \neg \psi_{n}\right) \\
& \text { (FLF3): } \bigwedge_{n \in\{1,2, \ldots m\}} \nabla_{n}
\end{aligned}
$$

For, given (FLF2), it would seem that we should conclude that for some $n \in\{1,2, \ldots m\}$ things would not be as the particular conjunct of (FLF1) claims they would be were $\phi_{n}$ to be the case. And so, given (FLF3), it would seem that (FLF1) and (FLF2) are inconsistent.

The intuition that claims of the form (FLF1) - (FLF3) are inconsistent is, furthermore, supported by some principled semantic theories of counterfactuals. ${ }^{10}$

\footnotetext{
${ }^{10}$ See Stalnaker [1968] and Lewis [1973].
} 
Claim: (FLF1) - (FLF3) are jointly inconsistent given Stalnaker's semantics for counterfactuals and given Lewis's semantics for counterfactuals.

Justification: We can think of Stalnaker's semantics for counterfactuals as the result of taking Lewis' semantics and imposing a restriction on the class of models. If, then, a set of claims is inconsistent given Lewis' semantics, it follows that it is also inconsistent given Stalnaker's semantics. We can focus, then, on Lewis's semantics.

According to Lewis' semantics, given a world $w$, there is a total pre-order on worlds $\leq_{w}$, such that, if $\phi$ is true at some world, then $\phi \square \leftrightarrow \psi$ is true at $w$ just in case there is some $w^{\prime}$ such that $\phi$ holds at $w^{\prime}$ and $\phi \rightarrow \psi$ holds at each $w^{\prime \prime}$ such that $w^{\prime \prime} \leq_{w} w^{\prime}$

Let $w$, then, be an arbitrary world and assume that (FLF1) - (FLF3) all hold at $w$. Given Lewis' semantics, it follows from (FLF1) and (FLF3) that: (i) for each $n \in\{1,2, \ldots m\}$, there is some $w_{n}$ such that $\phi_{n}$ holds at $w_{n}$ and $\phi_{n} \rightarrow \psi_{n}$ holds for each $w^{\prime \prime}$ such that $w^{\prime \prime} \leq_{w} w_{n}$. And it follows from (FLF2) and (FLF3) that: (ii) there is some $w^{\prime}$ such that $\bigvee_{n \in\{1,2, \ldots m\}} \phi_{n}$ holds at $w^{\prime}$ and $\underset{n \in\{1,2, \ldots m\}}{\bigvee} \phi_{n} \rightarrow$ $\bigvee_{n \in\{1,2, \ldots m\}}\left(\phi_{n} \wedge \neg \psi_{n}\right)$ holds for each $w^{\prime \prime}$ such that $w^{\prime \prime} \leq w^{\prime}$. But, given (i) and (ii), it follows that there is some $w^{\prime}$ such that: (a) for some $n \in\{1,2, \ldots m\}$, $\phi_{n}$ holds at $w^{\prime}$, (b) for each $n \in\{1,2, \ldots m\}, \phi_{n} \rightarrow \psi_{n}$ holds at $w^{\prime}$, and (c) $\bigvee_{n \in\{1,2, \ldots m\}} \phi_{n} \rightarrow \bigvee_{n \in\{1,2, \ldots m\}}\left(\phi_{n} \wedge \neg \psi_{n}\right)$ holds at $w^{\prime}$.

But no world that can satisfy conditions (a) - (c). Thus (FLF1) - (FLF3) cannot hold at $w$.

Now (ILF1) - (ILF3) are simply the respective infinite generalizations of (FLF1) (FLF3). And it seems to me that the same sorts of grounds that were adduced in support of the claim that instances of (FLF1) - (FLF3) are inconsistent may be adduced in support of the claim that instances of (ILF1) - (ILF3) are inconsistent. Thus just as it would seem that, given (FLF2), for some $n \in\{1,2, \ldots m\}$, things would not be as the particular conjunct of (FLF1) claims they would be were $\phi_{n}$ to be the case, so too does it seem that, given (ILF2), for some $n \in \mathbb{N}$, things would not be as the particular conjunct of (ILF1) claims they would be were $\phi_{n}$ to be the case.

Moreover, there are principled semantic theories of counterfactuals that entail the inconsistency of (ILF1) - (ILF3).

Claim: (ILF1) - (ILF3) are jointly inconsistent given Stalnaker's semantics for counterfactuals and given Lewis's semantics for counterfactuals together with the Limit Assumption.

Justification: If a set of claims are inconsistent given Lewis's semantics for counterfactuals together with the Limit Assumption, then they will also be in- 
consistent given Stalnaker's semantics. We can focus, then, on Lewis's semantics with the Limit Assumption.

The Limit Assumption tells us that, if $\phi$ holds at some world, then, for any world $w$, there will be some world $w^{\prime}$, at which $\phi$ holds, such that there is no $w^{\prime \prime}$ at which $\phi$ holds, such that $w^{\prime \prime} \leq_{w} w^{\prime}$ while $w^{\prime} \not_{w} w^{\prime \prime}$. That is, there will be a set of minimal $\phi$ permitting worlds given the pre-order $\leq_{w}$.

Let $w$, then, be an arbitrary world and let us assume that (ILF1) - (ILF3) hold at $w$. Given (ILF3) and the Limit Assumption, there will be a set of $\leq_{w}$ minimal worlds at which $\bigvee_{n \in \mathbb{N}} \phi_{n}$ holds. Let this set be C. Given (ILF2), we have that every $w^{\prime} \in \mathbf{C}$ is such that that $\bigvee_{n \in \mathbb{N}}\left(\phi_{n} \wedge \neg \psi_{n}\right)$ holds at $w^{\prime}$. Pick an arbitrary $w^{\prime} \in \mathbf{C}$ and let $m$ be such that $\phi_{m} \wedge \neg \psi_{m}$ holds at $w^{\prime}$. Then it follows that $\phi_{m} \square \leftrightarrow \psi_{m}$ cannot hold at $w$. For $w^{\prime}$ will be amongst the $\leq_{w}$ minimal worlds at which $\phi_{m}$ holds. But in order for $\phi_{m} \square \rightarrow \psi_{m}$ to hold at $w, \psi_{m}$ must hold at all such worlds. It follows, then, that (ILF1) does not hold at $w$, which contradicts our assumption that (ILF1) - (ILF3) hold at $w$.

There seems to me, then, good reason to say that claims of the form (ILF1) - (ILF3) are jointly inconsistent. Intuitively they would seem to be, and this intuition can be supported by principled semantic theories.

As we've seen, though, given that (ILF1) - (ILF3) are jointly inconsistent, there is a simple resolution to the paradox raised Benardete's scenario. For, given the joint inconsistency of (ILF1) - (ILF3), we must reject Intention Realization Possibility. Despite appearances, there simply is no possible world in which each of the gods is such that it would realize its intention were it called upon to do so. This is ruled out on principled logical grounds.

Indeed, in light of the plausibility of Conditional Compossibility, I'm inclined to see the paradox raised by Benardete's scenario as providing additional support for the claim that (ILF1) - (ILF3) are jointly inconsistent. For given this inconsistency, we have a simple resolution of the paradox, while without this principle it is hard to see how we can avoid rejecting the otherwise plausible principle Conditional Compossibility.

In closing, let me note one way in which the preceding may be brought to bear on a contested issue concerning the logic and semantics of counterfactuals. One principle that separates Lewis' semantics for counterfactuals and Stalnaker's is the Limit Assumption. Stalnaker accepts this principle, while Lewis rejects it. We've seen though that if you add the Limit Assumption to Lewis' semantics, then claims of the form (ILF1) - (ILF3) come out jointly inconsistent. It turns out, though, that if you give up the Limit Assumption such claims need not be inconsistent. Indeed, we can show the following. 
Claim: Intention Realization, Intention Failure, and Possible Passage are logically consistent, given Lewis's semantics for counterfactuals without the Limit Assumption.

Justification: Here's a model in which these three claims all come out true given Lewis' semantics. We have a world $w$ and, for each $n \in \mathbb{N}$, a world $w_{n}$. We assume that, for each $n, m \in \mathbb{N}$, if $m>n$, then $w_{m} \leq_{w} w_{n}$ and $w_{n} \not_{w} w_{m}$. We further assume that, for each $n \in \mathbb{N}, P_{\frac{1}{n+1}}$ and $\left(W_{\frac{1}{n}} \wedge \forall_{z \leq n} \neg P_{\frac{1}{z}}\right)$ hold at $w_{n}$, while for each $m>n, P_{\frac{1}{m}}$ and $\neg\left(W_{\frac{1}{n}} \wedge \forall_{z \leq n} \neg P_{\frac{1}{z}}\right)$ also hold at $w_{n}$.

If, then, one endorses Lewis' semantics for counterfactuals and rejects the Limit Assumption, it follows that one can't resolve the paradox raised by Benardete's scenario by claiming that, on logical grounds, Intention Realization must fail to hold. Now there are some wellknown counterintuitive consequences of combining Lewis' semantics with a rejection of the Limit Assumption. ${ }^{11}$ I suggest that Benardete's scenario can be seen as highlighting another such implausible consequence. It is, at the very least, a point in favor of accepting the Limit Assumption that, in doing so, one can provide a simple and principled resolution to the paradox raised by Benardete's scenario that is not available if one rejects this principle.

\section{References}

Jose Benardete. Infinity: An Essay in Metaphysics. Clarendon, 1964.

John Hawthorne. Before-effect and zeno causality. Nous, 34(4):622-633, 2000.

David Lewis. Counterfactuals. Harvard University Press, Cambridge, MA, 1973.

John L. Pollock. Subjunctive Reasoning. Dordrecht Reidel, 1976.

Graham Priest. On a version of one of zeno's paradoxes. Analysis, 59(1):1-2, 1999.

Robert Stalnaker. A theory of conditionals. In NIcholas Rescher, editor, Studies in Logical Theory. Blackwell, 1968.

Robert Stalnaker. Inquiry. MIT Press, 1984.

Gabriel Uzquiano. Before-effect without zeno causality. Nous, 46(2):259-264, 2012.

Stephen Yablo. A reply to new zeno. Analysis, 60(2):148-151, 2000.

\footnotetext{
${ }^{11}$ For some discussion see Lewis [1973] chapter 1, Stalnaker [1984] chapter 7, and Pollock [1976] chapter 1.
} 